\title{
AKAP9 Upregulation Predicts Unfavorable Prognosis in Pediatric Acute Myeloid Leukemia and Promotes Stemness Properties via the Wnt/ $\beta$-Catenin Pathway
}

\author{
Shiwen $\mathrm{Wu}$ iD ${ }^{1,2}$ \\ Dongqin Shen ${ }^{3}$ \\ Li Zhao ${ }^{4}$ \\ 'The First Clinical Medical College, \\ Lanzhou University, Lanzhou, Gansu, \\ People's Republic of China; ${ }^{2}$ Departments \\ of Clinical Laboratory, The First Hospital of \\ Lanzhou University, Lanzhou University, \\ Lanzhou, Gansu, People's Republic of \\ China; ${ }^{3}$ Department of Medical Oncology, \\ The First Hospital of Lanzhou University, \\ Lanzhou, Gansu, People's Republic of \\ China; ${ }^{4}$ Department of Central Laboratory, \\ Gansu Key Laboratory of Genetic Study of \\ Hematopathy, The First Hospital of \\ Lanzhou University, Lanzhou, Gansu, \\ People's Republic of China
}

Background: PRKA kinase anchor protein 9 (AKAP9) is a scaffold protein involved in various cellular processes, including cell adhesion, proliferation, differentiation, and apoptosis. Although the oncogenic role of AKAP9 in solid tumors is well elucidated, the functions and mechanisms of AKAP9 in acute myeloid leukemia (AML) are still not understood.

Methods: We used the gene expression omnibus (GEO) database (GSE2191) to determine the mRNA expression of AKAP9 in the bone marrow of pediatric AML and healthy patients. We further used the therapeutically available research to generate effective treatments (TARGET) database to elucidate the relationship between AKAP9 expression and clinical outcomes in pediatric patients with AML. In addition, cell proliferation, cell cycle, apoptosis, RT-PCR, and Western blotting assays were applied to reveal the functions of AKAP9 and the underlying mechanisms of AKAP9 silencing in THP1 and HL60 cell lines.

Results: AKAP9 is overexpressed in the bone marrow of pediatric AML patients as compared with that of healthy patients. High expression of AKAP9 was found to be a predictor of poor overall survival (OS) and event-free survival (EFS). Using univariate and multivariate survival analyses, we found that high AKAP9 expression is an independent predictor of a worse OS and EFS. Functionally, AKAP9 silencing significantly inhibited AML cell proliferation, and cell cycle progression and promoted apoptosis. Moreover, AKAP9 silencing significantly downregulated the expression of stemness markers and $\beta$ catenin.

Conclusion: AKAP9 upregulation is a predictor of unfavorable prognosis, promotes stemness, and activates the Wnt/ $\beta$-catenin pathway in AML patients. AKAP9 may act as a prognostic biomarker of AML in pediatric patients and a future therapeutic target.

Keywords: PRKA kinase anchor protein 9, acute myeloid leukemia, prognosis, leukemic stem cells, pediatric

\section{Introduction}

Acute myeloid leukemia (AML) is a common hematological malignancy characterized by a clonal malignant proliferation of myeloid primordial cells. ${ }^{1}$ AML has a poor prognosis and is the main cause of death in children and adults under 35 years of age in China. ${ }^{2}$ Chemotherapy remains the primary treatment option for patients with AML. Despite the improvement in AML research and therapies, 
several challenges still exist in the clinical treatment of this disease. ${ }^{3}$ Although the majority of patients achieve the stage of initial remission after induction chemotherapy, a large proportion of cases relapse and fail the reinduction chemotherapy. Accumulating evidence has suggested that leukemia stem cells (LSCs) play a key role in the recurrence and treatment failure of AML. ${ }^{4}$ Therefore, investigation of the mechanisms underlying the development and maintenance of LSCs and the development of more effective therapeutic targets of AML are essential. ${ }^{5}$

PRKA kinase anchor proteins (AKAPs) are a group of structurally diverse proteins, having the common function of binding to the regulatory subunit of protein kinase A (PKA) and limiting the holoenzyme to specific locales within cells. ${ }^{6}$ AKAP9 is a member of the AKAPs family, and its overexpression is implicated in solid cancer progression. ${ }^{7}$ But, the function of AKAP9 and its potential molecular mechanisms in hematology neoplasms, particularly AML, are unclear.

The purpose of the present study was to investigate AKAP9 expression in AML and elucidate the relationship between AKAP9 mRNA expression and the clinical prognosis of pediatric patients with AML. Additionally, we explored the function and potential molecular mechanisms involved in AKAP9-mediated progression and recurrence of AML.

\section{Materials and Methods}

\section{Patients and Samples}

The public microarray data of pediatric AML and normal control (pediatric healthy) were obtained from the gene expression omnibus (GEO) database (GSE2191) in May 2021. ${ }^{8}$ The differential expression of AKAP9 mRNA between 54 pediatric AML patients and 4 normal controls was analyzed by GEO2R. Moreover, we analyzed complete clinical and expression data of $285 \mathrm{AML}$ pediatric patients obtained from the therapeutically available research to generate effective treatments (TARGET, https://ocg.cancer.gov/programs/target/) database. Patients were categorized into High-AKAP9 and LowAKAP9 groups based on the median expression levels of AKAP9. We evaluated the correlation between mRNA expression of AKAP9 and prognosis and prognostic factors in patients with AML. The risk stratification was defined as the level of potential clinical risk based on the cytogenetic characteristics or molecular alterations as being related to those of acute myeloid leukemia. ${ }^{1}$
Univariate and multivariate COX analyses were constructed to identify the prognostic risk factors for overall survival (OS) and event-free survival (EFS) in pediatric patients with AML.

\section{Cell Culture}

Human AML cell lines (HL-60, THP1) were obtained from the cell bank of type culture collection of the Chinese academy of sciences (Shanghai, China) and cultured under standard conditions in RPMI-1640 media (Meilunbio, Dalian, China) supplemented with $1 \%$ penicillin-streptomycin and $10 \%$ fetal calf serum. The cells were incubated at $37^{\circ} \mathrm{C}$ in a humidified atmosphere with $5 \% \mathrm{CO}_{2}$.

\section{Lentivirus Infection}

To determine the biological functions of AKAP9 in AML cells, we conducted AKAP9 silencing experiments. AML cells were infected with lentivirus containing either a short hairpin RNA for AKAP9 (sh-AKAP9) or a negative control vector (sh-NC) according to the manufacturer's instructions. We observed green fluorescence at $48 \mathrm{~h}$ after lentivirus transfection using a fluorescence microscope (magnification, 100x). Puromycin was used for screening of the infected cells at $72 \mathrm{~h}$ after transfection. The silencing efficiencies of AKAP9 were identified by real-time quantitative polymerase chain reaction (RT-PCR) and Western blot analysis. shRNAs were synthesized by Genechem (Shanghai, China). The sequence of the AKAP9 shRNA used was 5'.GCCAAGAAGAAAGATTGATT TAAATCAATCTTTCTTCTTGGC. ${ }^{\prime}$.

\section{RNA Isolation and Real-Time Quantitative Polymerase Chain Reaction}

The total RNA was extracted using TRIzol reagent. The target cDNA was obtained by reverse transcription using a FastKing gDNA Dispelling RT SuperMix kit (TIANGEN, Beijing, China) according to the manufacturer's instructions. A SuperReal PreMix Plus kit (TIANGEN, Beijing, China) was used to perform RTPCR with specific primers as previously described. ${ }^{7,9}$ Primers for RT-PCR were synthesized by TaKaRa Biotechnology (Dalian, China). Each sample was tested on triplicates, and the mRNA expression was measured by the threshold cycle (CT) values. The relative expression of 
mRNA was calculated by the $2^{\Delta \Delta C T}$ method, and $\beta$-actin was used as an internal reference for mRNAs.

\section{Protein Isolation and Western Blot Analysis}

Total protein was extracted from the cells with RIPA buffer. The concentration of protein was determined by the BCA protein assay kit (MeiLunBio, Dalian, China). Equal amounts of protein were separated by SDS-PAGE and transferred onto the polyvinyl difluoride (PVDF) membranes. The membranes were blocked for $2 \mathrm{~h}$ with $5 \%$ non-fat milk in tris-buffered saline (TBS) at room temperature and incubated with primary antibodies against AKAP9 (1:500 dilution, Abcam, catalog ab32679), $\beta$ catenin (1:1000 dilution, Proteintech, catalog 66379. 1-Ig), CD133 (1:1000 dilution, Proteintech, 18470-1-AP), ALDH1A1 (1:1000 dilution, Proteintech, 15910-1-AP), and $\beta$-actin (1:1000 dilution, Proteintech, catalog 660091-Ig) at $4^{\circ} \mathrm{C}$ overnight. The membranes were then washed with TBS-tween-20 thrice and incubated with anti-mouse or anti-rabbit secondary antibodies (1:5000 dilution, Proteintech, catalog SA00001-1, and SA00001-2) for 2 $\mathrm{h}$ at room temperature. The protein bands were treated with ECL reagent (Beyotime Biotechnology, Shanghai, China) and visualized by Amersham Imager 600 visualizer (GE Healthcare, Pittsburgh, USA). The protein expression level was quantified and analyzed using ImageJ software (National Institutes of Health) software.

\section{Cell Proliferation Assays}

Cell proliferation ability was evaluated using cell counting kit-8 (CCK8) (MeiLunBio, Dalian, China) according to the manufacturer's protocol. First, the cells were inoculated into 96-well plates at a density of $5 \times 10^{4} /$ well and treated with CCK- 8 solution at $0,24,48,72$, and 96 h. Finally, the absorbance of each well was measured by a microplate spectrophotometer at $450 \mathrm{~nm}$.

\section{Cell Cycle Assays}

For cell-cycle analysis, the cells were washed with cold PBS, and then fixed with $70 \%$ ethanol at $4^{\circ} \mathrm{C}$ overnight. The cells were washed twice with PBS and incubated with $1 \mathrm{mg} / \mathrm{mL}$ propidium iodide and $0.5 \mathrm{mg} / \mathrm{mL}$ RNase A (MultiSciences Biotech, Co., Ltd., Hangzhou, China) for $30 \mathrm{~min}$ in dark at $37^{\circ} \mathrm{C}$. The phase change of the cell cycle was examined using a flow cytometer (BD Biosciences, San Jose, CA, USA). The flow cytometry data were analyzed using ModFit LT software for Windows (Version 5.0.9). All experiments were conducted in independent triplicates.

\section{Cell Apoptosis Analysis}

Annexin V-phycoerythrin (APC) and 7-aminoactinomycin D (7-AAD) apoptosis detection kits (MultiSciences Biotech, Co., Ltd., Hangzhou, China) were used to detect the apoptosis of THP1 and HL60 cells after AKAP9 silencing according to the manufacturer's protocols. In short, $5 \times 10^{5}$ cells were washed twice with cold PBS and resuspended in $500 \mu \mathrm{L}$ binding buffer. Next, the cells were stained with $5 \mu \mathrm{L}$ Annexin V-APC and $5 \mu \mathrm{L}$ 7-AAD for $15 \mathrm{~min}$ in the dark at room temperature. The apoptotic cell percentage was calculated using a flow cytometer (BD Biosciences, San Jose, CA, USA). The flow cytometry data were analyzed by FlowJo (version 10.7.1) software.

\section{Statistical Analysis}

Statistical analyses were carried out using SPSS 22.0 software (IBM, Armonk, New York, USA). OS and EFS were evaluated by the Kaplan-Meier method and analyzed using the Log rank test. Univariate and multivariate analyses of prognostic factors in patients with AML were conducted using the Cox regression model. Multivariate analysis included variables with $\mathrm{P}<0.1$ in univariate analysis. Pearson Chi-square and Fisher's exact tests were used for categorical variables. Quantitative variables were expressed as mean $\pm \mathrm{SD}$. The Student's $t$-test was used to determine differences between the two groups. A P-value less than 0.05 was considered statistically significant.

\section{Results AKAP9 Expression in Pediatric AML}

To explore the role of AKAP9 in AML pathogenesis, we evaluated the expression of AKAP9 in AML patients and normal control based on the GSE2191 from the GEO database, which contained 4 normal controls and 54 pediatric patients with AML. Differences in AKAP9 expression levels between pediatric AML and the normal group were evaluated using the Mann-Whitney $U$-test. The findings revealed that AKAP9 has a significantly higher expression in pediatric patients with AML compared to normal control $(\mathrm{P}<0.01)$ (Figure 1A).

To explore whether AKAP9 expression might play a role in relapse and drug resistance of AML in pediatric patients, 

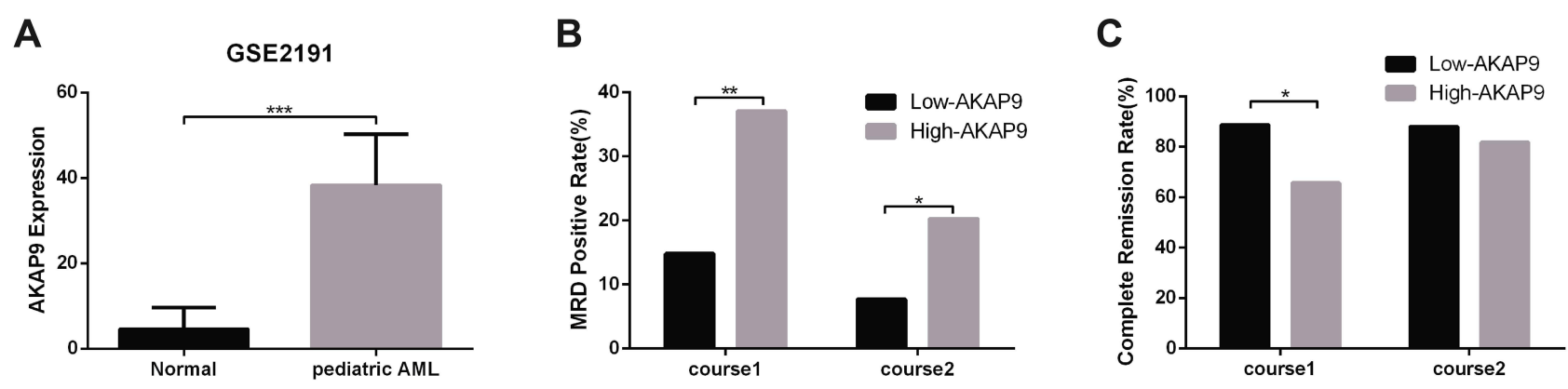

D

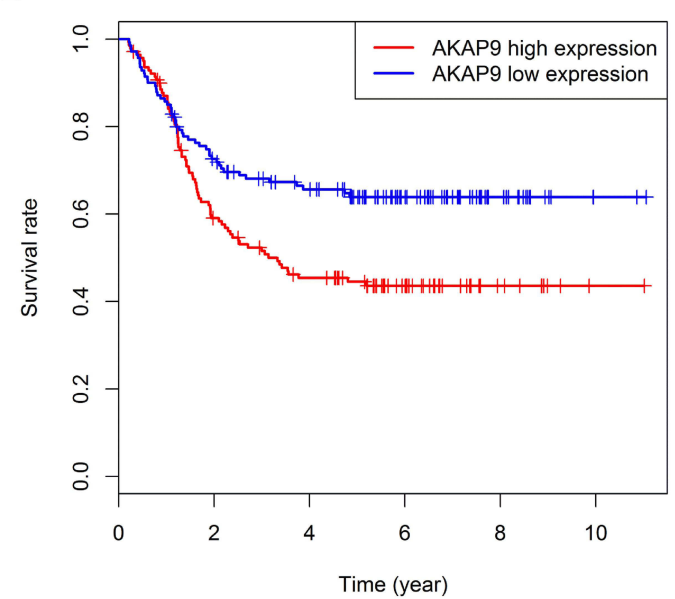

E

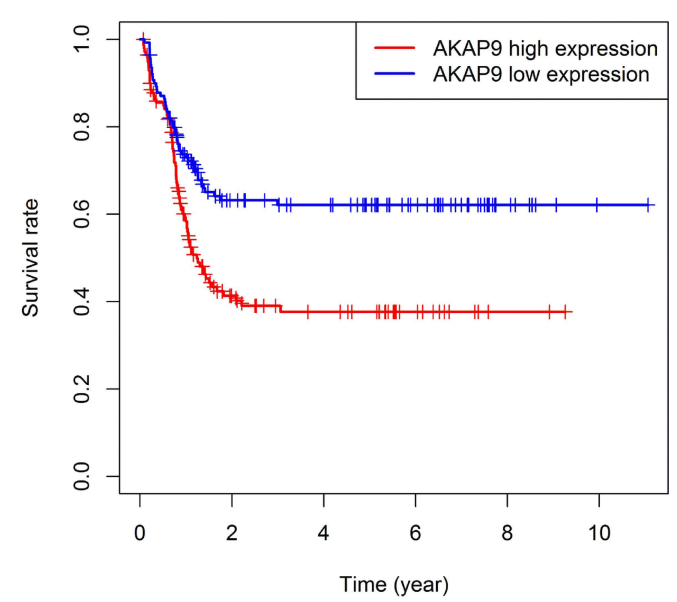

Figure I The AKAP9 mRNA expression and prognosis in pediatric patients with AML. (A) The AKAP9 mRNA expression level in pediatric AML and control in the GSE2191 cohort. (B) Patients with a high AKAP9 expression had a significantly higher MRD positive rate when compared with those with a low AKAP9 expression. (C) Patients with a high AKAP9 expression showed significantly higher CR rate when compared with those with a low AKAP9 expression. (D and E) High AKAP9 mRNA expression proved to be a predictor of poor overall survival and event-free survival, as ascertained from the TARGET database. $* \mathrm{P}<0.05$, $* * \mathrm{P}<0.0 \mathrm{I}$, ***P $<0.00 \mathrm{I}$.

we compared the positive rate of minimal residual disease (MRD) and complete remission (CR) rate between high- and low-AKAP9-expression, respectively, at end of the first and second course of chemotherapy. We found that the MRD rate in the high-AKAP9 group was significantly higher than that in the low-AKAP9 group at end of the first course (37.1 vs $14.8 \% ; \mathrm{P}<0.01$ ) and second course (20.3 vs $7.7 \%$; $\mathrm{P}<0.01$ ) of chemotherapy (Figure 1B). The CR rate in the low-AKAP9 group was significantly higher than that in the high-AKAP9 group (88.7\% vs $65.7 \%, \mathrm{P}<0.01)$ at end of the first course of chemotherapy; however, no significant differences were observed at the end of the second course of chemotherapy in patients with low and high AKAP9 expression ( 88.0 vs $81.8 \% ; \mathrm{P}=0.163$ ) (Figure $1 \mathrm{C}$ ).

\section{AKAP9 Overexpression is a Predictor of Poor Prognosis in Pediatric AML Patients} To determine the applicability of AKAP9 in the prognosis of pediatric AML, we analyzed the relationship between the $A K A P 9$ expression level and OS/EFS time based on the TARGET database. We found that patients with high AKAP9 expression had a shorter OS and EFS time compared to patients with low AKAP9 expression (Figure 1D and E). These findings suggest that the high expression of AKAP9 can result in poor survival in pediatric patients with AML. Therefore, AKAP9 can act as a significant biomarker to determine the prognosis of pediatric AML patients.

\section{Association Between AKAP9 Expression and Clinical Characteristics of Pediatric AML}

We investigated the association between the AKAP9 expression and the clinicopathological features of pediatric patients with AML. Patients with low AKAP9 expression showed higher white blood cell counts than those with high AKAP9 expression (median, $59.0 \times 10^{9} / \mathrm{L}$ vs $31.1 \times$ $\left.10^{9} / \mathrm{L} ; \mathrm{P}<0.01\right)$. Significant differences were observed in 
Table I Comparison of Clinical Manifestations and Laboratory Features Between Low-AKAP9 and High-AKAP9 AML Patients

\begin{tabular}{|c|c|c|c|}
\hline Characteristics & $\begin{array}{c}\text { LowAKAP9 } \\
(n=142)\end{array}$ & $\begin{array}{l}\text { HighAKAP9 } \\
\qquad(n=\mid 43)\end{array}$ & $P$ \\
\hline $\begin{array}{l}\text { Age/years, median } \\
\text { (range) }\end{array}$ & $\mathrm{II}(0-22)$ & $9(0-23)$ & 0.202 \\
\hline Gender, no. (\%) & & & 0.438 \\
\hline Male & $79(55.63)$ & $73(51.05)$ & \\
\hline Female & $63(44.37)$ & $70(48.95)$ & \\
\hline Race, no. (\%) & & & 0.840 \\
\hline Caucasian & $105(73.94)$ & $105(73.43)$ & \\
\hline African American & $16(11.27)$ & $16(11.19)$ & \\
\hline Asian & $3(2.11)$ & $6(4.20)$ & \\
\hline Other & $6(4.23)$ & $7(4.90)$ & \\
\hline Unknown & $12(8.45)$ & $9(6.30)$ & \\
\hline $\begin{array}{l}\text { WBC at diagnosis/ } \\
\times 10^{9} / \mathrm{L} \text {, median (range) }\end{array}$ & $59.0(1.6-446)$ & $31.1(0.9-519)$ & $<0.01$ \\
\hline $\begin{array}{l}\text { BM blast/ \%, median } \\
\text { (range) }\end{array}$ & $77.5(31-100)$ & $72.0(14-100)$ & 0.009 \\
\hline $\begin{array}{l}\text { PB blast } / \% \text {, median } \\
\text { (range) }\end{array}$ & $66.0(0-97)$ & $52.0(0-97)$ & $<0.01$ \\
\hline Risk stratification, no. (\%) & & & 0.044 \\
\hline Low & $67(47.18)$ & $47(32.87)$ & \\
\hline Standard & $51(35.92)$ & $74(51.75)$ & \\
\hline High & $18(12.68)$ & $15(10.49)$ & \\
\hline Unknown & $6(4.23)$ & $7(4.90)$ & \\
\hline Karyotype, no. (\%) & & & 0.677 \\
\hline Normal & $34(23.94)$ & $37(25.87)$ & \\
\hline Abnormal & $101(7|| 3)$. & $96(67.13)$ & \\
\hline Unknown & $7(4.93)$ & $10(6.99)$ & \\
\hline FLT3-ITD, no. (\%) & & & 0.894 \\
\hline Positive & $23(16.20)$ & $24(16.78)$ & \\
\hline Negative & $119(83.80)$ & $119(83.22)$ & \\
\hline
\end{tabular}

Abbreviations: WBC, white blood cells; BM, bone marrow; PB, peripheral blood; FLT3-ITD, internal tandem duplication of the FLT3 gene.

the bone marrow $(\mathrm{BM})$ blast percentages $(\mathrm{P}=0.009)$, peripheral blood $(\mathrm{PB})$ blast percentages $(\mathrm{P}<0.01)$, and risk stratification $(\mathrm{P}=0.044)$ between the two groups. No significant relationships were noted between the AKAP9 expression and age, gender, race, karyotype status, and internal tandem duplication of the FLT3 (FLT3-ITD) (Table 1).

\section{AKAP9 Overexpression is Independently Associated with Poor Prognosis of Pediatric AML Patients}

To investigate whether AKAP9 is an independent prognostic factor in AML, we conducted univariate and multivariate Cox analysis for OS and EFS using the TARGET database. The univariate analysis showed that three factors - FLT3-ITD, risk stratification, and AKAP9 expression - are risk markers associated with shorter OS, while four factors - FLT3-ITD, risk stratification, FAB category, and AKAP9 expression - are risk markers associated with shorter EFS. As per the findings of multivariate analysis, AKAP9 overexpression is an independent prognostic factor for poor OS and EFS in pediatric AML patients (Figure 2A and B).

\section{Establishment of AKAP9 Stable Down-Regulation AML Cell Line}

To investigate the biological functions of AKAP9 in AML, THP1, and HL60 cells were infected with lentivirus containing AKAP9-shRNA to conduct stable knock-down of AKAP9. As shown in Figure 3A, the cells that were successfully transfected with lentivirus showed green fluorescence after $48 \mathrm{~h}$. The RT-PCR and Western blot results revealed that the mRNA and protein levels of AKAP9 in THP1 and HL60 cells infected with lentivirus containing AKAP9-shRNA were significantly downregulated as compared with the corresponding levels in the control cells $(\mathrm{P}<0.001$; Figure 3B-D).

\section{AKAP9 Silencing Inhibits Cell Proliferation and Cell Cycle Progression of AML Cells}

CCK8 assays were performed to evaluate the effect of AKAP9 silencing on cell proliferation in AML cells. According to the growth curve, the proliferation of THP1 cells and HL60 cells was significantly suppressed after AKAP9 silencing, while cells transfected with shNC exhibited no significant inhibition of cell proliferation (Figure 4A and B).

To explore whether AKAP9 is involved in the cell cycle of AML cells, we assessed the effect of AKAP9 silencing on the cell cycle distribution of THP1 cells and HL60 cells using FACS. The findings suggested that AKAP9 silencing slightly, but statistically significantly, prolonged the $\mathrm{G} 0 / \mathrm{G} 1$ phase $(5.38 \%$ and $3.87 \%)$ and reduced the $\mathrm{G} 2 / \mathrm{M}$ phase $(4.14 \%$ and $4.91 \%)$ in both THP1 and HL60 cells; however, the S phase was not significantly changed. As expected, the G0/G1 phase, $\mathrm{S}$ phase, and G2/M phase were not changed in sh-NC transfected cells (Figure 4C-F). Together, these findings revealed that AKAP9 gene silencing significantly decreases the cell proliferation potential and significantly inhibits the cell cycle progression of AML cells. 
A

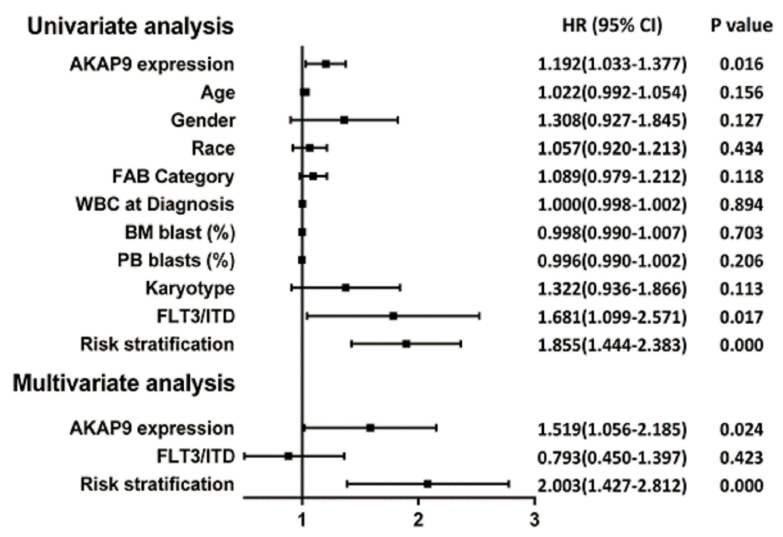

B

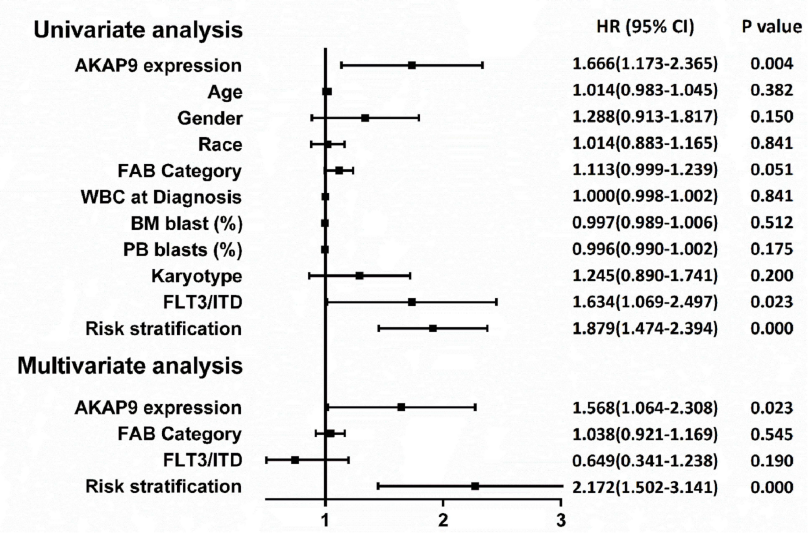

Figure 2 Univariate and multivariate COX analyses of prognostic factors for OS and EFS in pediatric patients with AML. (A) The AKAP9 overexpression is an independent prognostic factor of worse OS in pediatric patients with AML. (B) AKAP9 overexpression is an independent prognostic factor of worse EFS in pediatric patients with AML. Abbreviations: $\mathrm{Cl}$, confidence interval; $\mathrm{HR}$, hazard ratio.

A

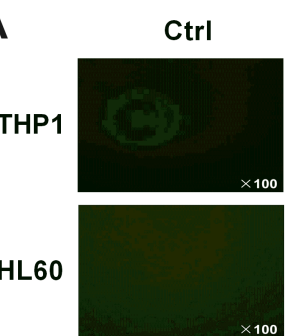

C

Ctrl sh-NC sh-AKAP9 Ctrl sh-NC sh-AKAP9

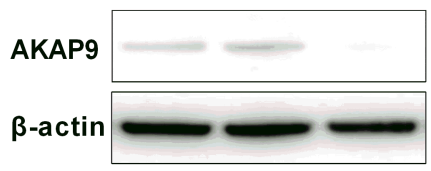

THP1
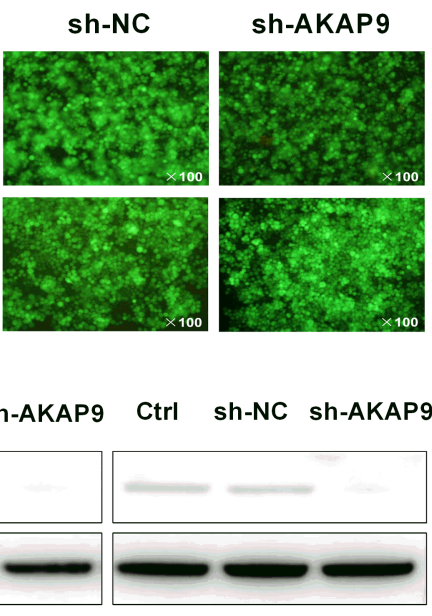

HL60
B
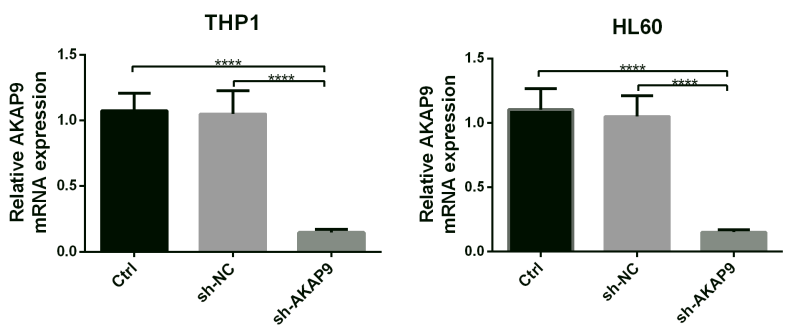

D

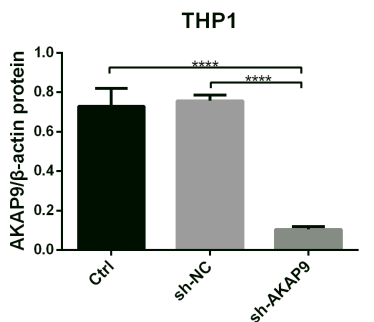

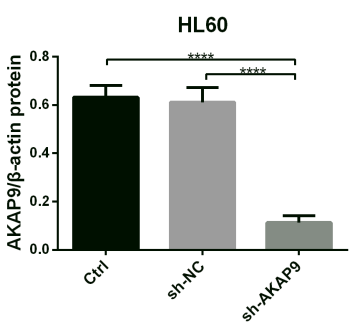

Figure 3 Establishment of AKAP9-silenced THPI and HL60 cell lines. (A) Fluorescence images of THPI and HL60 cells at $48 \mathrm{~h}$ after transfection. (B) The mRNA and (C) protein expression levels of AKAP9 in THPI and HL60 were reduced in AKAP9 silenced-cell lines. (D) Quantitative analysis of the AKAP9 protein expression in (C). ****P $<$ 0.0001 .

\section{AKAP9 Silencing Promotes the Apoptosis of AML Cells}

We determined whether AKAP9 is involved in AML cell apoptosis. Annexin V APC/7-AAD double-staining assays revealed that the average percentage of apoptotic cells in the sh-AKAP9 group (THP1, 11.23\%; HL60, 11.61\%) was significantly higher than the corresponding percentage in sh-NC (THP1, 7.15\%; HL60, 5.52\%) and control (THP1, 7.52\%; HL60, 5.80\%) groups $(\mathrm{P}<0.01$, Figure $5 \mathrm{~A}$ and $\mathrm{B}$ ). There were no significant differences in the apoptotic rates between the control and sh-NC groups $(\mathrm{P}>0.05$; Figure $5 \mathrm{~B})$. The results revealed that
AKAP9 silencing significantly promotes apoptosis in THP1 and HL60 cells.

\section{AKAP9 Silencing Decreased Stem Cell Marker Expression of AML Cells}

To further investigate the effect of AKAP9 on the stemness of AML cells, we analyzed the stem cell biomarkers including ALDH1A1 and CD133 by Western blot. The expression levels of ALDH1A1 in THP1 and HL60 cells were significantly decreased after AKAP9 silencing (Figure 6A-C). These data suggest that AKAP9 might be a regulator of AML stem cell phenotype. 
A

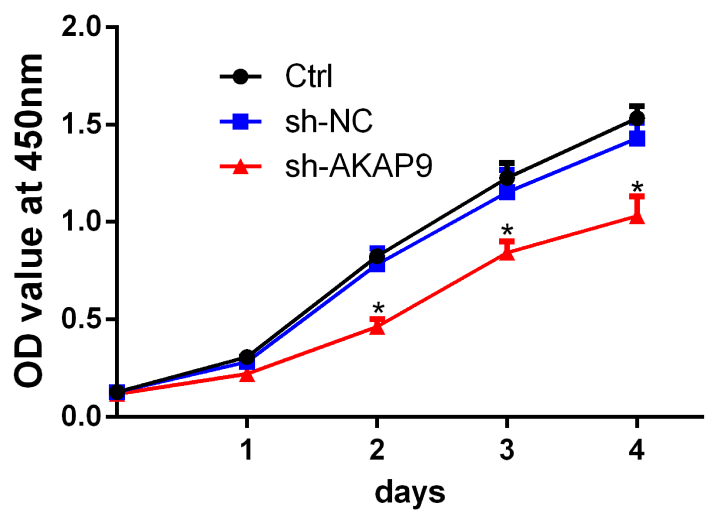

C

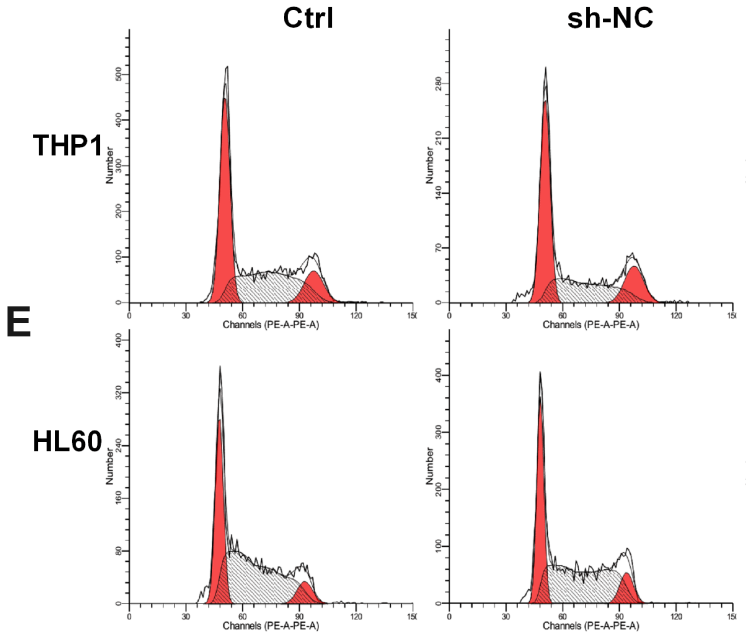

B

HL60

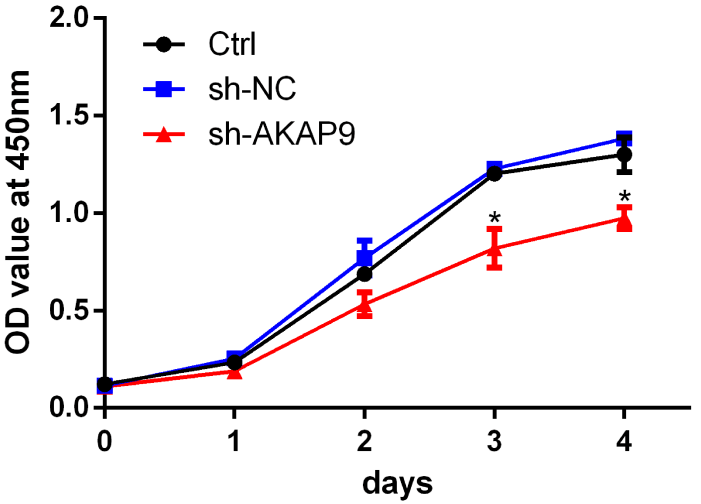

D

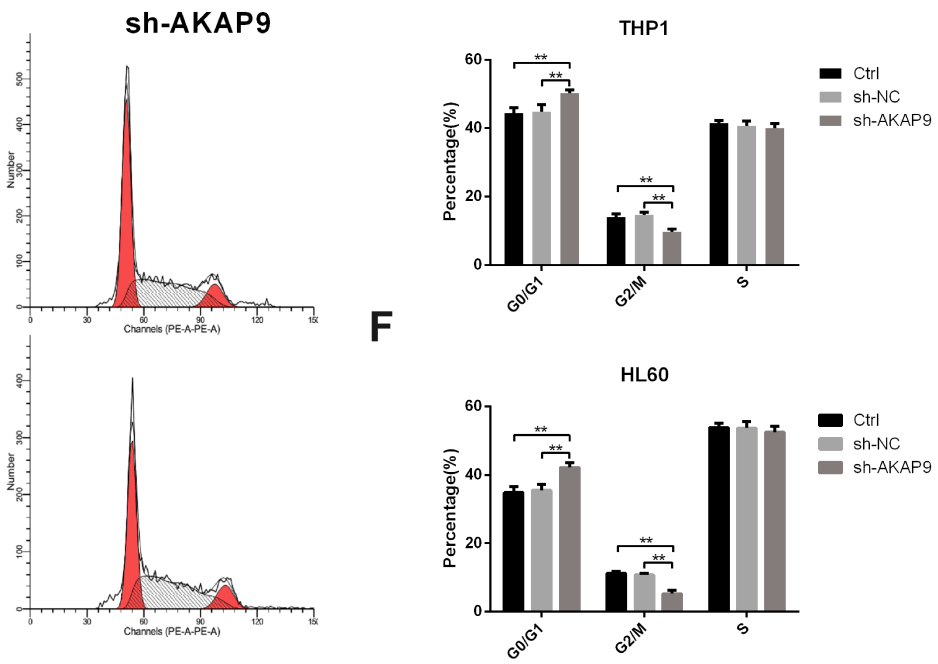

Figure 4 Effects of AKAP9 silencing on the proliferation and cell cycle of THPI and HL60 cells. (A and B) AKAP9 silencing decreased the potential of THPI and HL60 cells' proliferation. (C and D) and (E and F) AKAP9 silencing inhibited the cell cycle progression of THPI and HL60 cells. $* \mathrm{P}<0.05$, $* * \mathrm{P}<0.0 \mathrm{I}$.

A

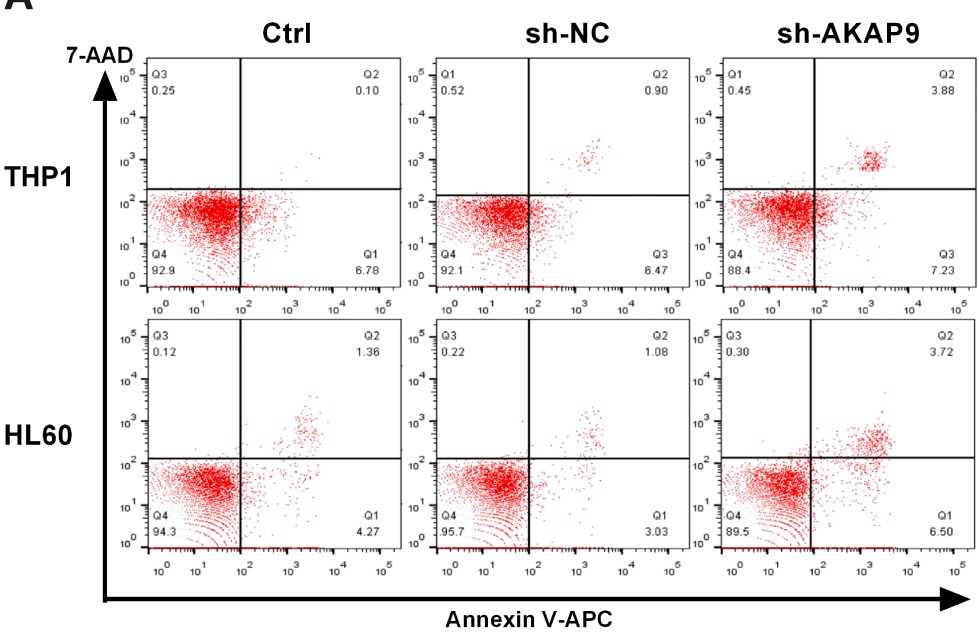

B

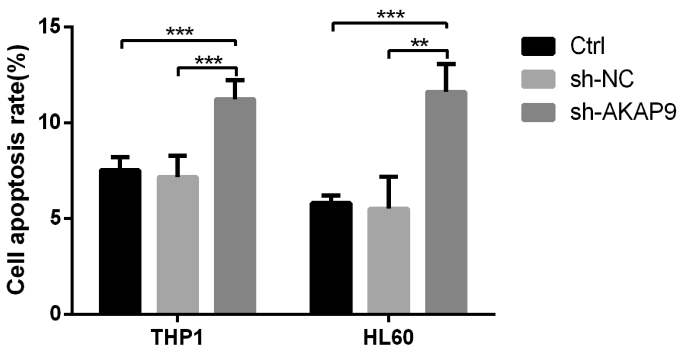

Figure 5 AKAP9 silencing promoted the apoptosis of HL60 and THPI cells. (A) Apoptotic cells were analyzed by flow cytometry by staining annexin APC/7-AAD. (B) The percentage of apoptosis in THPI and HL60 cells after AKAP9 silencing. **P $<0.01$, ***P $<0.00$ I. 


\section{AKAP9 Silencing Suppresses the Wnt/ $\beta$-catenin Signaling Pathway in AML Cells}

The $\mathrm{Wnt} / \beta$-catenin signaling pathway is commonly involved in the stemness of cancer cells. The inhibition of $\mathrm{Wnt} / \beta$ catenin signaling can reduce the stemness of AML cells. To determine whether AKAP9 participates in regulating the stemness of AML cells via Wnt/ $\beta$-Catenin signaling, we evaluated the relationship between AKAP9 and $\beta$-catenin (CTNNB1), which is a key regulatory molecule of the Wnt signaling pathway. The mRNA expression level of CTTNB1 in AKAP9-high patients was significantly higher than that in AKAP9-low patients (Figure 7A). We also observed a significant correlation between AKAP9 and $\beta$-catenin mRNA expressions $(\mathrm{P}<0.001)$ (Figure $7 \mathrm{~B})$. Furthermore, Western blot assays were conducted to determine the expression of AKAP9 and intracellular signal transducer $\beta$-catenin in AKAP9-modulating cells. The results showed that AKAP9 down-regulation suppressed the expression of $\beta$ catenin (Figure $7 \mathrm{C}$ and D). All these findings suggested that AKAP9 silencing inhibits the Wnt/ $\beta$-Catenin signaling pathway in AML cells.

\section{Discussion}

AKAP9, also known as Yotiao, is linked to the development and metastasis of several human cancers,

A

Ctrl sh-NC sh-AKAPg

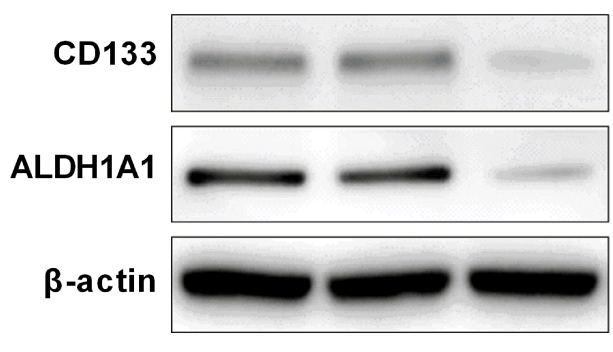

THP1

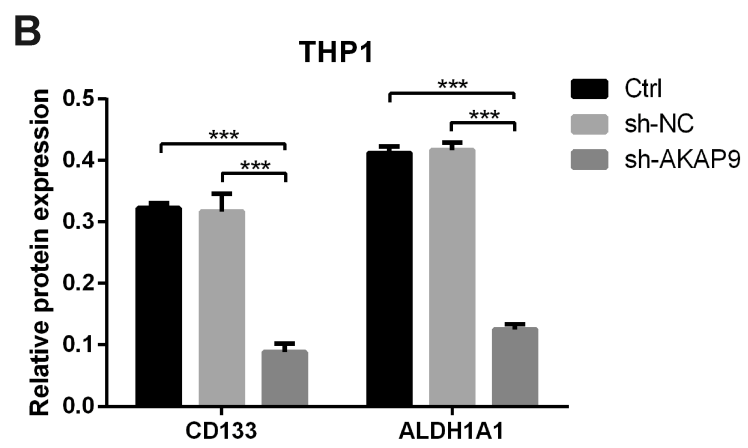

including lung cancer, ${ }^{10}$ breast cancer, ${ }^{11,12}$ colorectal cancer, ${ }^{7,13}$ melanomas, ${ }^{14}$ thyroid carcinomas, ${ }^{15,16}$ bladder cancer, ${ }^{17}$ and oral cancer. ${ }^{18}$ However, the functions and mechanisms of AKAP9 in these tumors are not yet completely elucidated. Also, there are no available demonstrating the role of AKAP9 in the development of AML. Our results suggested that AKAP9 expression is upregulated in pediatric AML patients, and its high expression is associated with poor prognosis. AKAP9 overexpression was found to be independently associated with poor prognosis of pediatric AML patients. AKAP9 plays an important role in the progression and relapse of AML; however, the related underlying mechanisms remain poorly understood.

LSCs are the subset of cells exhibiting quiescent and low energy production state and possess limitless selfrenewal properties. ${ }^{19}$ The development of treatment strategies targeting LSCs is expected to facilitate the development of a more effective cure for AML. It is unclear whether AKAP9 plays a critical role in LSCs. AKAP9 is the key regulator in the $\mathrm{G} 1$ to $\mathrm{S}$ phase transition. ${ }^{20}$ Our cell experiments confirmed that AKAP9 silencing could inhibit the growth of AML cells and regulate the cell cycle progression. MRD is a significant predictor of AML recurrence and prognosis. MRD positive status

\section{Ctrl sh-NC sh-AKAP9}

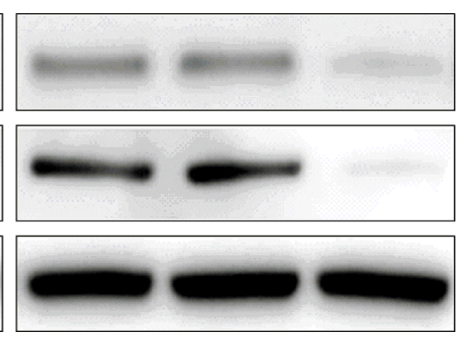

HL60

Figure 6 Effect of AKAP9 silencing on the stem cell-related molecular markers in HL60 and THPI cells. (A) Western blotting of stem cell-related molecular markersALDHIAI and CDI33-in the control (Ctrl), sh-NC-, and sh-AKAP9-transfected THPI and HL60 cells. (B and C) Densitometry analyses of the AKAP9 protein expression levels in THPI and HL60. $* * * P<0.001$.

C

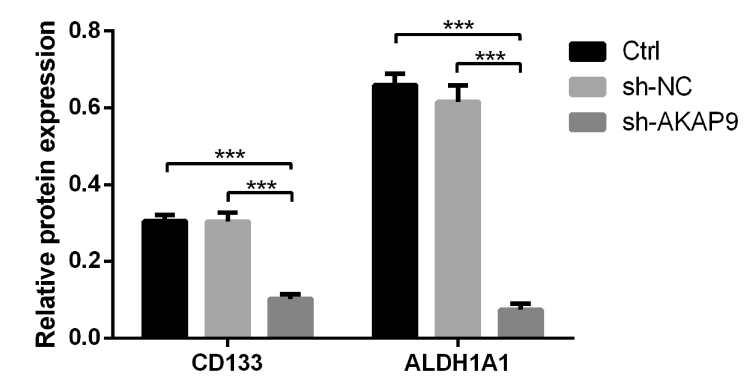


A

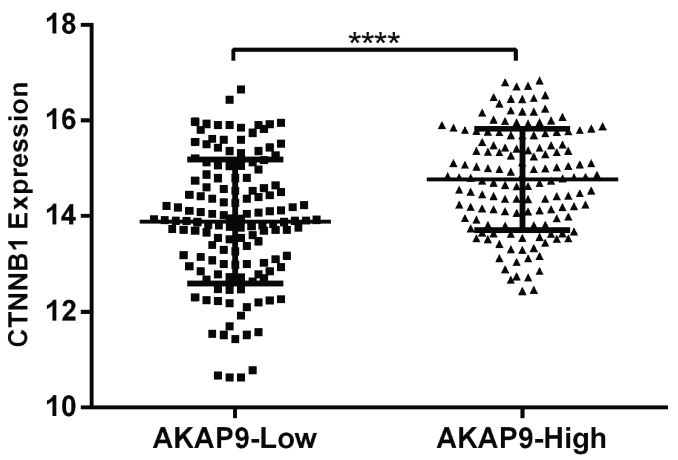

C

Ctrl sh-NC sh-AKAP9 Ctrl sh-NC sh-AKAPg

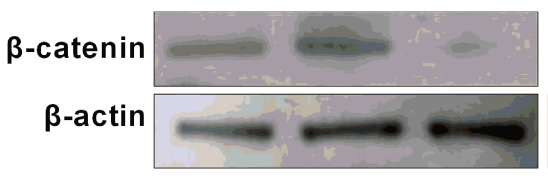

THP1

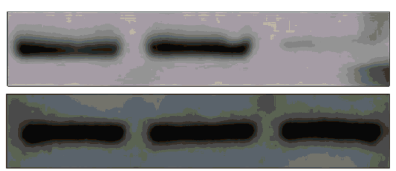

HL60
B

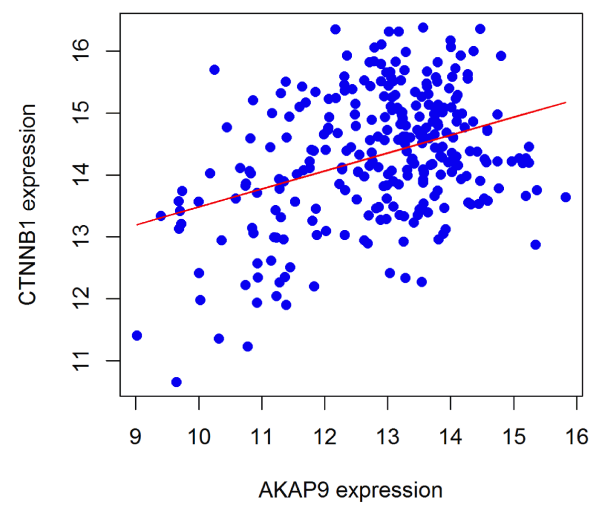

D

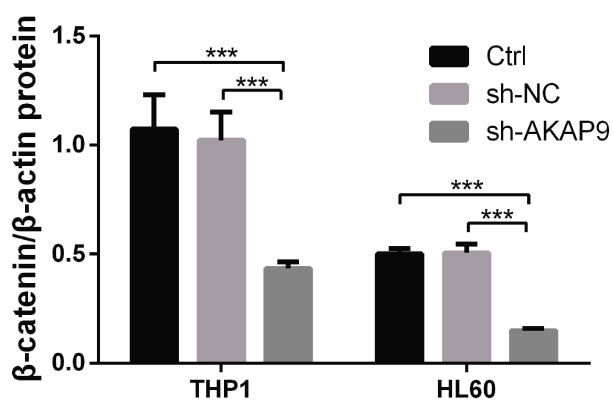

Figure 7 AKAP9 silencing suppressed the Wnt/B-catenin signaling pathway in AML cells. (A) The mRNA expression level of CTTNBI in AKAP9-high and AKAP9-low patients from the TARGET database. (B) The correlation of CTTNBI and AKAP9 mRNA expressions in pediatric AML patients from the TARGET database. (C) Western blotting of $\beta$-catenin in the control (Ctrl), sh-NC-, and sh-AKAP9-transfected THPI and HL60 cells. (D) Densitometry analysis of the $\beta$-catenin protein expression levels in (C). ${ }^{*} * \mathrm{P}<0.001$, $* * * * \mathrm{P}<0.0001$.

indicated that a small amount of LSC remained in the bone marrow protective environment even after the complete remission of chemotherapy (or stem cell transplantation). ${ }^{21,22}$ Patients with high AKAP9 expression had a higher positivity rate of MRD compared with patients with low AKAP9 expression. Furthermore, ALDH1A1 and CD133 are important markers in LSCs. $^{23,24}$ ALDH1A1 plays an important role in initiation, relapse, chemotherapy resistance, and progression of AML. These processes are involved in the prognosis of AML. ${ }^{25-27}$ Moreover, CD133 expression is highly associated with chemoresistance and prognosis of patients with AML. ${ }^{28}$ Our results showed that AKAP9 silencing decreases the expression of ALDH1A1 and CD133 in AML cells. The findings support the hypothesis that AKAP9 overexpression contributes to AML cell stemness.

The Wnt/ $\beta$-catenin pathway is at the center of carcinogenesis and cancer progression. It is necessary for the maintenance and self-renewal of LSCs. $\beta$-catenin is considered a vital downstream effector of the $\mathrm{Wnt} / \beta$-catenin pathway. $^{29,30}$ Several molecules involved in the $\mathrm{Wnt} / \beta$ - catenin pathway have a prognostic value in AML. ${ }^{29}$ Therapeutics targeting the Wnt/ $\beta$-catenin pathway have a great potential to treat AML. Studies have shown that the AKAP gene family is involved in the $\mathrm{Wnt} / \beta$-catenin pathway. The AKAP family members can not only bind to $\beta$-catenin but also directly interact with GSK3beta, which is required for the regulation of $\beta$-catenin and thus for Wnt signaling $^{31-33}$ Our results reveal that AKAP9 and $\beta$ catenin mRNA expressions are highly correlated, and AKAP9 silencing suppresses the protein levels of $\beta$ catenin, indicating that AKAP9 may promote the activation of $\beta$-catenin and $\mathrm{Wnt} / \beta$-catenin signaling pathway.

\section{Conclusion}

In conclusion, AKAP9 mRNA expression is upregulated in pediatric AML patients, and its expression influences patient prognosis. AKAP9 silencing significantly inhibits AML cell proliferation and cell cycle progression and promotes apoptosis. Moreover, AKAP9 increases LSCs stemness by activating the $\mathrm{Wnt} / \beta$-catenin signaling pathway. AKAP9 may serve as a prognostic indicator for pediatric $\mathrm{AML}$ and provide a novel molecular drug target 
for AML. Further in vivo experiments and patient-derived sample studies are needed to validate our findings.

\section{Abbreviations}

AKAP9, A-kinase anchor proteins 9; FAB category, French-American-British classification category; FLT3ITD, internal tandem duplication of the FLT3 gene; EFS, event-free survival; OS, overall survival; TARGET, the therapeutically available research to generate effective treatments database; GEO, the gene expression omnibus database.

\section{Ethical Statement}

The present study was approved by the Ethics Committee of LZU No. 1 Hospital.

\section{Acknowledgments}

This work was supported by the University Innovation Fund Project of Gansu Educational Committee (2021B017) and the Foundation of the First Hospital of Lanzhou University (Grant no. ldyyyn2019-33).

\section{Disclosure}

The authors report no conflicts of interest in this work.

\section{References}

1. Dohner H, Estey E, Grimwade D, et al. Diagnosis and management of AML in adults: 2017 ELN recommendations from an international expert panel. Blood. 2017;129(4):424-447. doi:10.1182/blood-201608-733196

2. Wang N, Feng YJ, Wang BH, et al. Disease burden of leukemia in the Chinese population, in 1990 and 2013. Zhonghua Liu Xing Bing Xue Za Zhi. 2016;37(6):783-787. doi:10.3760/cma.j.issn.02546450.2016.06.008

3. Dohner H, Weisdorf DJ, Bloomfield CD, Longo DL. Acute myeloid leukemia. $N$ Engl J Med. 2015;373(12):1136-1152. doi:10.1056/ NEJMra1406184

4. Thomas D, Majeti R. Biology and relevance of human acute myeloid leukemia stem cells. Blood. 2017;129(12):1577-1585. doi:10.1182/ blood-2016-10-696054

5. Pollyea DA, Jordan CT. Therapeutic targeting of acute myeloid leukemia stem cells. Blood. 2017;129(12):1627-1635. doi:10.1182/blood2016-10-696039

6. Schmidt PH, Dransfield DT, Claudio JO, et al. AKAP350, a multiply spliced protein kinase A-anchoring protein associated with centrosomes. J Biol Chem. 1999;274(5):3055-3066. doi:10.1074/ jbc.274.5.3055

7. Hu ZY, Liu YP, Xie LY, et al. AKAP-9 promotes colorectal cancer development by regulating $\mathrm{Cdc} 42$ interacting protein 4 . Biochim Biophys Acta. 2016;1862(6):1172-1181. doi:10.1016/j. bbadis.2016.03.012

8. Yagi T, Morimoto A, Eguchi M, et al. Identification of a gene expression signature associated with pediatric AML prognosis. Blood. 2003;102(5):1849-1856. doi:10.1182/blood-2003-02-0578
9. Sheng Y, Yu C, Liu Y, et al. FOXM1 regulates leukemia stem cell quiescence and survival in MLL-rearranged AML. Nat Commun. 2020;11(1):928. doi:10.1038/s41467-020-14590-9

10. Truong T, Sauter W, McKay JD, et al. International lung cancer consortium: coordinated association study of 10 potential lung cancer susceptibility variants. Carcinogenesis. 2010;31(4):625-633. doi:10.1093/carcin/bgq001

11. Frank B, Wiestler M, Kropp S, et al. Association of a common AKAP9 variant with breast cancer risk: a collaborative analysis. J Natl Cancer Inst. 2008;100(6):437-442. doi:10.1093/jnci/djn037

12. Kumar N, Gupta S, Dabral S, Singh S, Sehrawat S. Role of exchange protein directly activated by cAMP (EPAC1) in breast cancer cell migration and apoptosis. Mol Cell Biochem. 2017;430(1-2):115-125. doi:10.1007/s11010-017-2959-3

13. Yang MH, Hu ZY, Xu C, et al. MALAT1 promotes colorectal cancer cell proliferation/migration/invasion via PRKA kinase anchor protein 9. Biochim Biophys Acta. 2015;1852(1):166-174. doi:10.1016/j. bbadis.2014.11.013

14. Kabbarah O, Nogueira C, Feng B, et al. Integrative genome comparison of primary and metastatic melanomas. PLoS One. 2010;5(5): e10770. doi:10.1371/journal.pone.0010770

15. Caria P, Vanni R. Cytogenetic and molecular events in adenoma and well-differentiated thyroid follicular-cell neoplasia. Cancer Genet Cytogenet. 2010;203(1):21-29. doi:10.1016/j. cancergencyto.2010.08.025

16. Lee JH, Lee ES, Kim YS, Won NH, Chae YS. BRAF mutation and AKAP9 expression in sporadic papillary thyroid carcinomas. Pathology. 2006;38(3):201-204. doi:10.1080/00313020600696264

17. Han Y, Zheng Q, Tian Y, Ji Z, Ye H. Identification of a nine-gene panel as a prognostic indicator for recurrence with muscle-invasive bladder cancer. J Surg Oncol. 2019;119(8):1145-1154. doi:10.1002/ jso. 25446

18. Onken MD, Winkler AE, Kanchi KL, et al. A surprising cross-species conservation in the genomic landscape of mouse and human oral cancer identifies a transcriptional signature predicting metastatic disease. Clin Cancer Res. 2014;20(11):2873-2884. doi:10.1158/1078-0432.CCR-14-0205

19. Wang Y, Krivtsov AV, Sinha AU, et al. The Wnt/beta-catenin pathway is required for the development of leukemia stem cells in AML. Science. 2010;327(5973):1650-1653. doi:10.1126/science.1186624

20. Mattaloni SM, Ferretti AC, Tonucci FM, Favre C, Goldenring JR, Larocca MC. Centrosomal AKAP350 modulates the G1/S transition. Cell Logist. 2013;3(1):e26331. doi:10.4161/c1.26331

21. Gomez-Arteaga A, Guzman ML. Minimal residual disease in acute myeloid leukemia. Adv Exp Med Biol. 2018;1100:111-125. doi:10.1007/978-3-319-97746-1_7

22. Chen X, Cherian S. Role of minimal residual disease testing in acute myeloid leukemia. Clin Lab Med. 2021;41(3):467-483. doi:10.1016/ j.cll.2021.03.017

23. Fleischman AG. ALDH marks leukemia stem cell. Blood. 2012;119 (15):3376-3377. doi:10.1182/blood-2012-02-406751

24. Heo SK, Noh EK, Ju LJ, et al. CD45(dim)CD34(+)CD38(-)CD133 $(+)$ cells have the potential as leukemic stem cells in acute myeloid leukemia. BMC Cancer. 2020;20(1):285. doi:10.1186/s12885-02006760-1

25. Hoang VT, Buss EC, Wang W, et al. The rarity of ALDH(+) cells is the key to separation of normal versus leukemia stem cells by ALDH activity in AML patients. Int $J$ Cancer. 2015;137(3):525-536. doi:10.1002/ijc. 29410

26. Yang X, Yao R, Wang H. Update of ALDH as a potential biomarker and therapeutic target for AML. Biomed Res Int. 2018;2018:9192104. doi:10.1155/2018/9192104

27. Nomura M, Rainusso N, Lee $\mathrm{YC}$, et al. Tegavivint and the beta-catenin/ALDH axis in chemotherapy-resistant and metastatic osteosarcoma. J Natl Cancer Inst. 2019;111(11):1216-1227. doi:10.1093/jnci/djz026 
28. Tolba FM, Foda ME, Kamal HM, Elshabrawy DA. Expression of CD133 in acute leukemia. Med Oncol. 2013;30(2):527. doi:10.1007/ s12032-013-0527-6

29. Gruszka AM, Valli D, Alcalay M. Wnt signalling in acute myeloid leukaemia. Cells. 2019;8(11):1403. doi:10.3390/cells8111403

30. Yang Y, Mallampati S, Sun B, et al. Wnt pathway contributes to the protection by bone marrow stromal cells of acute lymphoblastic leukemia cells and is a potential therapeutic target. Cancer Lett. 2013;333(1):9-17. doi:10.1016/j.canlet.2012.11.056

31. Li CC, Le K, Kato J, Moss J, Vaughan M. Enhancement of beta-catenin activity by BIG1 plus BIG2 via Arf activation and cAMP signals. Proc Natl Acad Sci $U$ S A. 2016;113 (21):5946-5951. doi:10.1073/pnas.1601918113
32. Gorski JA, Gomez LL, Scott JD, Dell'Acqua ML. Association of an A-kinase-anchoring protein signaling scaffold with cadherin adhesion molecules in neurons and epithelial cells. Mol Biol Cell. 2005;16 (8):3574-3590. doi:10.1091/mbc.e05-02-0134

33. Dema A, Schroter MF, Perets E, et al. The A-kinase anchoring protein (AKAP) glycogen synthase kinase 3beta interaction protein (GSKIP) regulates beta-catenin through its interactions with both protein kinase A (PKA) and GSK3beta. J Biol Chem. 2016;291 (37):19618-19630. doi:10.1074/jbc.M116.738047

\section{Publish your work in this journal}

Cancer Management and Research is an international, peer-reviewed open access journal focusing on cancer research and the optimal use of preventative and integrated treatment interventions to achieve improved outcomes, enhanced survival and quality of life for the cancer patient.
The manuscript management system is completely online and includes a very quick and fair peer-review system, which is all easy to use. Visit http://www.dovepress.com/testimonials.php to read real quotes from published authors. 